\title{
Fuzzy Comprehensive Evaluation Model for Road Capacity of Open Neighborhood -- Based on Improved NS Cellular Automata
}

\author{
Xiao-Yan Cao ${ }^{1}$, Bing-Qian Liu ${ }^{1}$, Jian $\mathrm{Cao}^{1} \&$ Yuan-Biao Zhang ${ }^{1,2,3}$ \\ ${ }^{1}$ Mathematical Modeling Innovative Practice Base, Jinan University, Zhuhai Campus, Zhuhai 519070, China \\ ${ }^{2}$ Packaging Engineering Institute, Jinan University, Zhuhai Campus, Zhuhai 519070, China \\ ${ }^{3}$ Key Laboratory of Product Packaging and Logistics of Guangdong Higher Education Institutes, Jinan University, \\ Zhuhai Campus, Zhuhai 519070, China \\ Correspondence: Yuan-Biao Zhang, Packaging Engineering Institute, Jinan University, Zhuhai Campus. E-mail: \\ zybt@jnu.edu.cn
}

Received: June 9, 2018

Accepted: June 25, $2018 \quad$ Online Published: June 30, 2018

doi:10.5539/mas.v12n7p144

URL: https://doi.org/10.5539/mas.v12n7p144

\begin{abstract}
With the rapid development of motorization in our country, gated communities have become the obstacle of the development of urban traffic network. In this paper, focusing on the impact of gated communities on the traffic situation in surrounding area before and after it is opened, we firstly establish a comprehensive evaluation system by fuzzy comprehensive evaluation method and then build up the improved NS model based on Cellular Automata, which put forward the concept of internal road sharing rate in communities. Finally, we select the urban district of different types and surrounding roads in Shanghai as a case, and through simulation and evaluation, we found that the opening of communities is feasible. What's more, compared with gated communities, the two-way 2-lane urban opening communities have the most optimal effect on improving road capacity.
\end{abstract}

Keywords: cellular automata, improved ns model, fuzzy comprehensive evaluation

\section{Introduction}

For a long time, due to the impact of historical factors and planning system, closed residential area has become the main form of China's urban residential area. With the rapid development of social economy and the continuous expansion of urban scale, the urban traffic congestion problem in China is increasingly severe. Closed residential area has destroyed the road network structure of urban, and blocked the city's "capillaries", which is an important factor of urban traffic congestion problems.

As the opening of closed residential area, Li Xiangpeng (2014) studied the closed-type residential areas suitable for opening based on the specific distance between the internal and external roads. Yu Yong (2006) studied the planning concept of "open blocks" in Europe and conducted a comparative analysis of urban housing construction in China. Pucher J (2003) studied the theory of "neighborhood units" in order to provide a residential community environment that is suitable for residents' lives, comfort, safety and complete facilities. Zhou and Bian (2000) demonstrated the process and existing problems of the development of the residential area model of China using the neighborhood unit as the prototype. Through the induction of the trend of foreign residential space construction since the 1960s, the author puts forward some suggestions on the construction of residential areas in China. Miao (2004) criticized the social problems arising from the closed residential area and proposed a planning model. Gao and Zhang (2006) analyzed the reasons for the popularization of the closed residential area in China and discussed its negative effects to the city. Li Jun (2007) thinks that the open community needs to grasp the appropriate level by analyzing the security, road system, and public facilities of closed and open communities. Qin (2010) studied the coordination between the closed residential area's planning models and motorization of the current urban, especially the rapid development of the car, and sought the balanced development between urban land planning and urban traffic development.

This paper studied the effect of the traffic capacity of the surrounding area after opening the closed residential area, and the effect of the open residential area is evaluated according to average passing speed, saturation, traffic density and accumulation of passing time. The paper studied the influence of different geographical types and different structures of surrounding road on the opening of the community in Shanghai. 


\section{Method}

\subsection{Evaluation Index of Traffic Capacity}

As an organic whole, the evaluation index should be able to reflect the dynamic changes of the traffic capacity and the traffic system of the surrounding roads in residential area from different aspects ( $\mathrm{Li} \& \mathrm{Zhu}$ et al, 2017). Therefore, this paper chooses four indexes, that is, average passing speed, saturation, traffic density and accumulation of passing time.

\subsubsection{Average Passing Speed}

Average passing speed refers to the average speed of all vehicles passing through the section of a highway, which reflects the smoothness of the section. The specific formula is as follows:

$$
v=\frac{1}{n} \sum_{i=1}^{n} v_{i}
$$

where, $v$ is the average speed (in $\mathrm{m} / \mathrm{s}$ ) of all vehicles passing through the section of a highway; $n$ is the equivalent number of vehicles(in pcu) within the road; $v_{i}$ is the average speed of the $i$-th car in the section of a $\operatorname{highway}(\mathrm{m} / \mathrm{s})$.

\subsubsection{Saturation}

Saturation is the ratio of actual traffic volume to traffic capacity, which reflects the extent of traffic congestion. The specific formula is as follows:

$$
P=\frac{V}{C}
$$

where, $P$ is saturation(in $\mathrm{pcu} / \mathrm{h}) ; V$ is the actual traffic volume within the $\operatorname{road}(\mathrm{in} \mathrm{pcu} / \mathrm{h}$ ); $C$ is the traffic capacity of the lane(in $\mathrm{pcu} / \mathrm{h}$ ).

In addition, the traffic capacity of the lane is calculated by

$$
C=s * \frac{g}{c}
$$

where, $C$ is the traffic capacity of the lane(in pcu/h);s is the saturation flow rate of the lane (in pcu/h); $g$ is the effective green time of the traffic lights (in s); $c$ is the cycle length of the traffic lights (in s).

Combine equations (1) and (2), the saturation can be defined by

$$
P=\frac{V^{*} c}{s^{*} g}
$$

\subsubsection{Traffic Density}

Traffic density refers to the number of vehicles on per unit length of a lane in a mount, which indicates the degree of concentration of the vehicle. The specific formula is as follows:

$$
K=\frac{n}{a^{*} L}
$$

where, $K$ is traffic density (in pcu/m); $n$ is the equivalent number of vehicles within the $\operatorname{road}(\mathrm{in} \mathrm{pcu}) ; a$ is the number of the lanes; $L$ is the length of the section of a highway(in $\mathrm{m}$ ).

\subsubsection{Accumulation of Passing Time}

Accumulation of Passing Time refers to the sum of the time of all vehicles on the road section, which reflects the congestion status of the section. The specific formula is as follows:

$$
T=\sum_{i=1}^{n}\left(T_{\text {iend }}-T_{\text {istart }}\right)
$$

Where, $T$ is accumulation of passing time; $n$ is the equivalent number of vehicles within the road; $T_{\text {istart }}$ is the moment of the $i$-th car entered the road; $T_{i e n d}$ is the moment of the $i$-th car driving out the road. 


\subsection{Comprehensive Evaluation Index}

Fuzzy comprehensive evaluation combines qualitative and quantitative factors to expand amount of information, so that the evaluation dimension to improve and the evaluation conclusion credible (Wang \& Liu, 2015). The influence of the traffic on surrounding road after opening residential area is a relatively complex fuzzy system. Based on the above evaluation index system, the fuzzy comprehensive evaluation can be used to evaluate it.

Step 1: Determine a set of factors to evaluate the traffic of the surrounding roads on the residential area $U$

$$
U=\left(u_{1}, u_{2}, u_{3}, u_{4}\right)
$$

Where, $u_{1}$ is average passing speed $v ; u_{2}$ is saturation $P ; u_{3}$ is traffic density $K ; u_{4}$ is accumulation of passing time $T$.

Step 2: Determine the strength level $V$

$$
V=\left(v_{1}, v_{2}, v_{3}, v_{4}, v_{5}\right)
$$

Where, $V=$ (strongest, stronger, strong, weak, weaker), the corresponding values are 5, 4, 3, 2, 1 .

Step 3: Evaluating the single factor to determine the fuzzy evaluation matrix

$$
R=\left(\begin{array}{llll}
r_{11} & r_{12} & \cdots & r_{15} \\
r_{21} & r_{22} & \cdots & r_{25} \\
\cdots & \cdots & \cdots & \cdots \\
r_{41} & r_{42} & \cdots & r_{45}
\end{array}\right), 0 \leq r_{i j} \leq 1
$$

Where, $r_{i j}$ is a subordinate relationship of $u_{i}$ in $U$ to $v_{i}$ in $V$.

In this paper, it can be considered that the large Cauchy distribution function is suitable as the membership function of the evaluation system. The specific formula is as follows:

$$
f(x)= \begin{cases}\frac{1}{1+\alpha(x-\beta)^{-2}}, & 1 \leq x \leq 3 \\ \eta \cdot \ln x+\phi, & 3 \leq x \leq 5\end{cases}
$$

Where, $\alpha, \beta, \eta, \phi$ are undetermined parameters.

According to the actual analysis, when evaluate the impact of the traffic on surrounding road after opening residential area, if the degree of influence is "strongest", then the membership degree is 0.9 ; if the degree of influence is "stronger", then the membership degree is 0.6 ; if the degree of influence is "weak", then the membership degree is 0.15 . According to this can use MATLAB to find the value of $\alpha, \beta, \eta, \phi$.

$$
[\alpha \beta \eta \phi]=\left[\begin{array}{lll}
114.06 & -2.56 & 1.34-1.26
\end{array}\right]
$$

Step 4: Determine the weight of evaluation

Substituting $\alpha, \beta, \eta, \phi$ into the large Cauchy distribution function, we can get the membership degree and the normalized result of each evaluation index. The results are shown in Table 1.

Table 1. The Membership Degree and the Normalized Result of Each Evaluation Index

\begin{tabular}{ccccc}
\hline Evaluation Index & $\begin{array}{c}\text { Average Passing } \\
\text { Speed }\end{array}$ & Saturation & Traffic Density & $\begin{array}{c}\text { Accumulation of } \\
\text { Passing Time }\end{array}$ \\
\hline $\begin{array}{c}\text { Intensity Values } \\
\text { Membership } \\
\text { Degree }\end{array}$ & stronger & weak & weak & strongest \\
& 0.6 & 0.15 & 0.15 & 0.9
\end{tabular}


Normalized Results

From Table 1, we can get the vector of weight of evaluation:

$$
A=\left[\begin{array}{llll}
0.333 & 0.083 & 0.083 & 0.500
\end{array}\right]
$$

\section{Step 5: Comprehensive Evaluation}

In order to weaken the impact of dimension and order of magnitude, to highlight the effect of the evaluation indicators, we need to pretreat the above four indicators.

\section{(1) Average Passing Speed}

Considering that the average passing speed will increase with the improvement of the traffic condition to a certain extent, we regard it as a greatest-type index. The specific formula is as follows:

$$
\Delta v=\frac{v_{\text {new }}-v_{0}}{v_{0}}
$$

Where, $v_{0}$ is the average passing speed in the initial state; $v_{\text {new }}$ is the average passing speed after the traffic change.

\section{(2) Saturation}

Saturation will be reduced with the improvement of traffic conditions to some extent, that is, the smaller the better, so it can be regarded as a minimal type indicator. The specific formula is as follows:

$$
\Delta p=\frac{p_{0}-p_{\text {new }}}{p_{0}}
$$

Where, $p_{0}$ is the saturation in the initial state; $p_{\text {new }}$ is the saturation after the traffic change.

(3) Traffic Density and Accumulation of Passing Time

Traffic density and accumulation of passing time will be reduced with the improvement of traffic conditions to some extent, that is, the smaller the better the indicators. They can be regarded as minimal type indicators, and the preprocessing formulas are similar to saturation.

In summary, we can get the comprehensive evaluation index (Score ). The specific formula is as follows:

$$
\text { Score }=0.333 \cdot \Delta v+0.083 \cdot \Delta P+0.083 \cdot \Delta K+0.500 \cdot \Delta T
$$

Score is also a greatest-type index, which is shows the overall traffic condition around the surrounding roads of the residential area. The greater the value, the stronger the traffic capacity of the road, that is, the traffic situation is improved.

\subsection{The Traffic Model of Roads around the Community}

In this paper, we study the influence of community opening on the surrounding road traffic, introduce the cellular automata model which can simulate various complicated traffic phenomena, and use the simulation results to quantify the traffic capacity of the vehicle, so as to evaluate the opening effect more effectively and objectively. A cellular automaton is a typical method for studying complex systems which is especially suitable for the study of spatiotemporal dynamics of complex systems. The essence is a dynamic system evolved over discrete time dimensions in a cell space with discrete and finite states according to certain local rules. In the field of vehicle traffic, cellular automata are widely used as representatives of microscopic models. The application is mainly divided into two categories: NS (or NaSch) model as the representative of the urban road traffic flow research; BML model as the representative of the urban transport network research (Peng, Wu \& Liu et al, 2015). This paper mainly studies the influence of community opening on the surrounding roads, mainly to the traffic flow, and weaken the structure of the traffic network, thus it is based on the NS model.

\subsubsection{NS Model}

The NS model makes the following abstracts for road traffic:

Using the one-dimensional discrete lattice chain which length is $L$ to simulate the road, as the cell space, we take 
$7.5 \mathrm{~m}$; Each grid represents a cell, at some point, the cell is empty or occupied by a vehicle; Array $X_{(n)}(t)$ indicates the position of the nth car at the $t$ moment of the grid point chain. Array $V_{(n)}(t)$ indicates the range of vehicle speed, $V_{\max }$ indicates the maximum speed,so the range of $V_{(n)}(t)$ is $0 \sim V_{\max }$, we take $V_{\max }=2$, which is the maximum speed is $54 \mathrm{~km} / \mathrm{h} . d_{(n)}$ indicates the spacing of adjacent vehicles, $d_{(n)}=X_{(n+1)}-X_{(n)}-1$

All vehicles are in the same direction (such as right).

$t$ time driving state: $V_{1}=1, V_{2}=2, V_{3}=1, V_{4}=0$

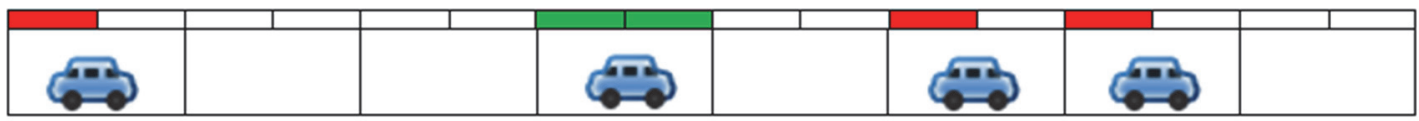

Figure 1. Initial state

Follow the rules below to update to the time $\mathrm{t}+1$

1) Acceleration process: $V_{n}=\min \left\{V_{\max }, V_{n}+1\right\}, V_{\max }=2$

If the distance is appropriate, then the rear of the vehicle will be accelerated, which simulate the reality of the driver's driving psychology;

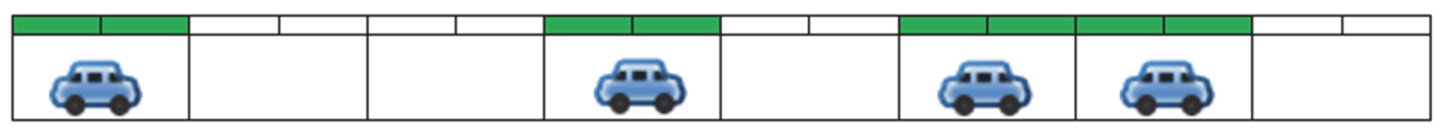

Figure 2. Acceleration process

2) Deceleration process: $V_{n}=\min \left\{V_{n}, d_{n}-1\right\}$

If the distance of the two cars before and after is too close, then the rear of the vehicle need to determine whether there will be a collision to the current speed, only consider slowing;

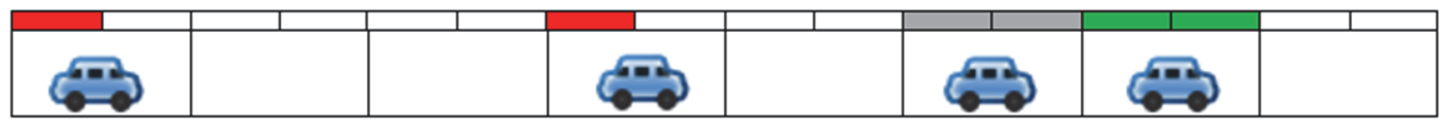

Figure 3. Deceleration process

3) Random slow process: $V_{n}=\max \left\{V_{n}-1,0\right\}, \operatorname{rand}_{1} \leq \beta$

The actual physiological - psychological factors of the proposed, due to various uncertainties, the vehicle may slow down;

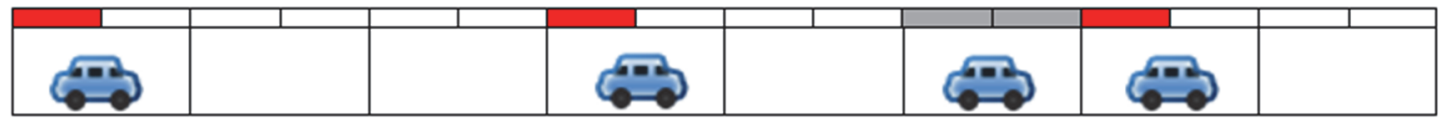

Figure 4. Slow process

4) Location update: $X_{n}=X_{n}+V_{n}$

Having considered the changes of current moment, update the location of the vehicle, showing the next moment of road traffic situation.

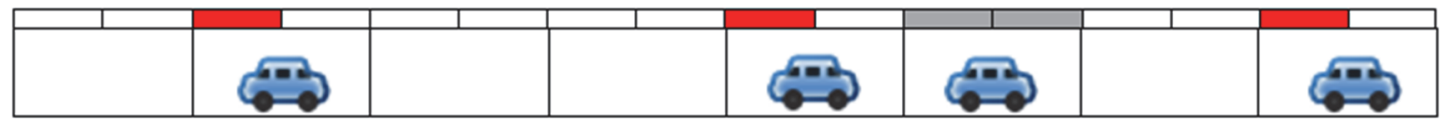

Figure 5. Location update

So far, we get the travel situation of vehicles at a certain moment on the road.

\subsubsection{The Exaltation of the Basic Mode of the Main Road}

The actual phenomenon of overtaking and the driver's ability to judge the road conditions will have a great impact 
on the state of the vehicle operation. In order to overcome the limitations of traditional NS model, this paper proposed the following lane overtaking rules combined with the driver's driving psychology ( $\mathrm{Li}, \mathrm{Qu} \& \mathrm{Liu}, 2016$ ).

Prepare for lane change

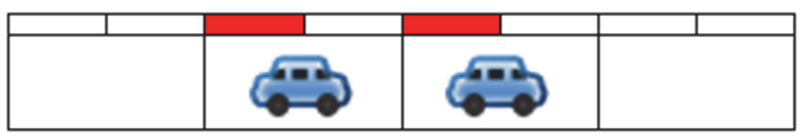

Figure 6. Prepare for lane change

If a car is followed by a car in front, it is a prerequisite for considering the lane change, and then considers the probability that the driver wishes to pass over the track, which sets to $\alpha$. According to experience, take $\alpha=0.8$.

Reversing conditions

a. According to the road traffic rules of motor vehicle, the driver priority to turn left when he lane overtaking. If there is no car on the left, then the driver will turn left.
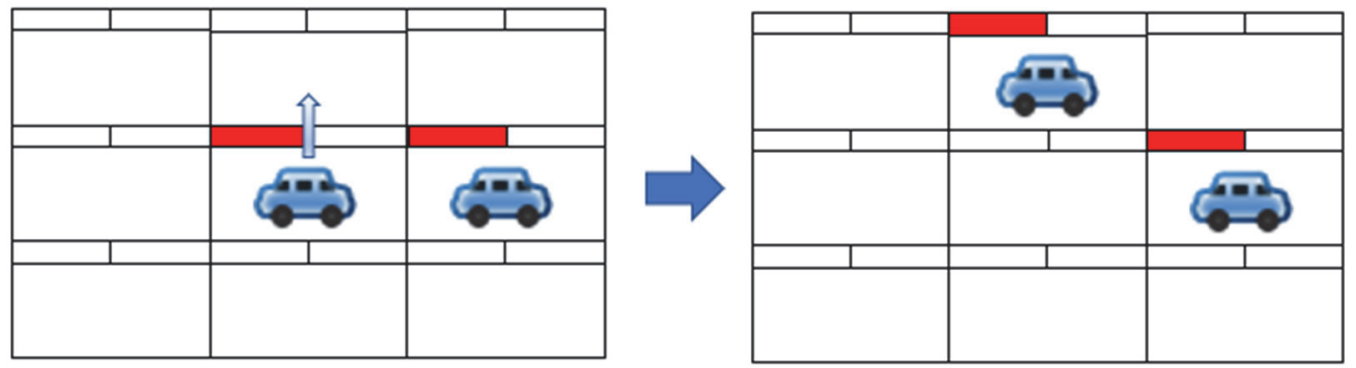

Figure 7. Turn left

b. If there is a car on the left, then determine whether there is a car on the right. If there is no car on the right, then the driver will change the right way.
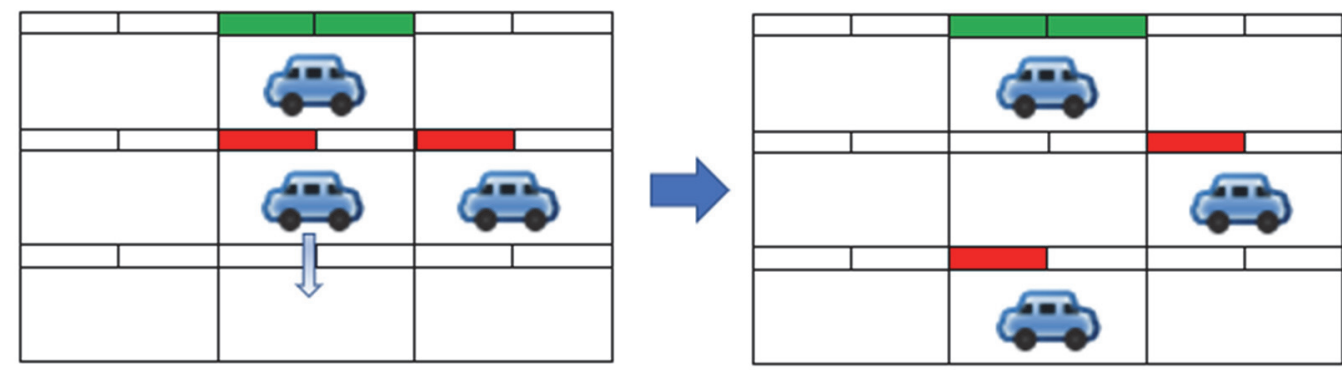

Figure 8. Turn right

c. If there is a car on both sides, then it is a failure.

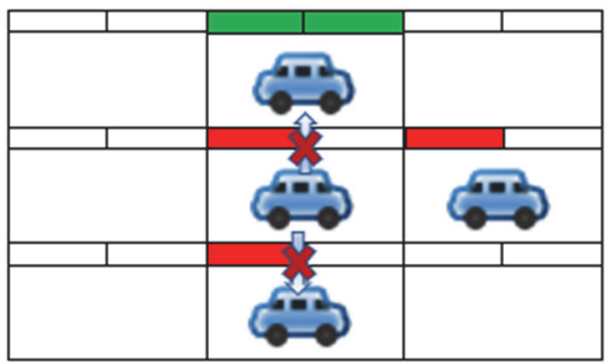

Figure 9. Lane failure 


\subsubsection{The Driving Mode of Community T-Shaped Intersection}

The paper study of the surrounding roads of the district, considering that the car within the district enter or leave the surrounding roads from the cell door, that is, produced a T-shaped intersection in the cell door, in order to prevent traffic congestion, we think that the vehicle to enter and exit the district can only turn right. Accordingly, this article introduces the T-type intersection access rules.

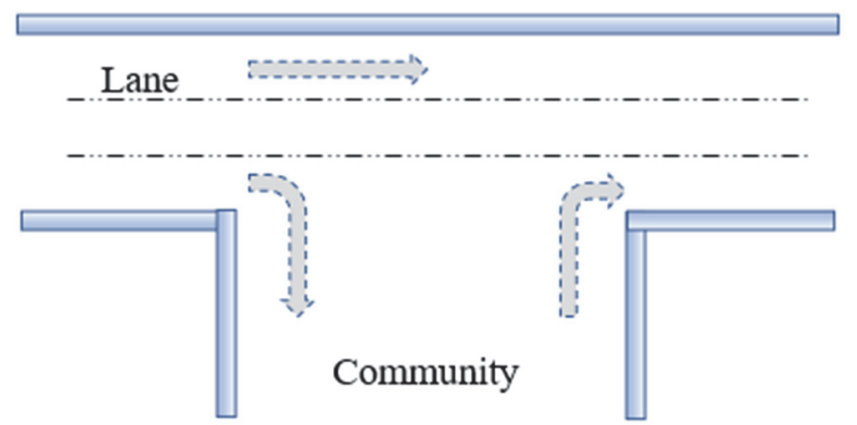

Figure 10. Traffic sketch map of T-junction

1) Enter the district

When the car arrival position $\mathrm{C}$, consider whether to enter the district, if you intend to enter the district, issue a right turn signal.

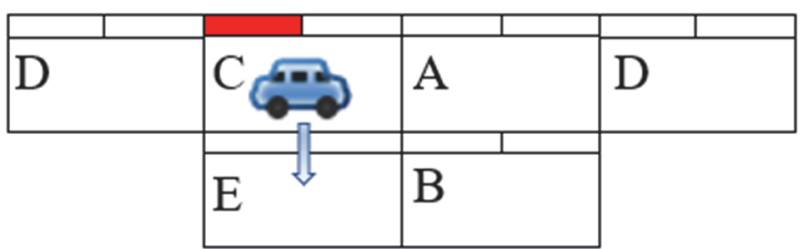

Figure 11. Into the district

In the position $\mathrm{C}$ of the vehicle, the states of the surrounding road after enter the district.

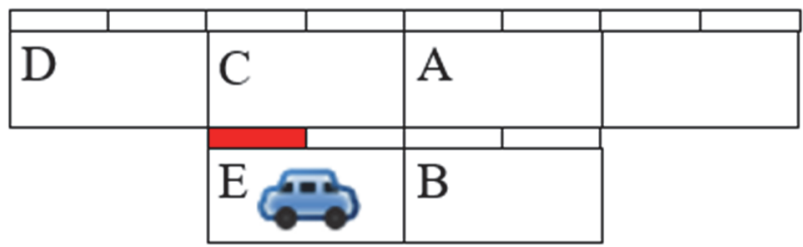

Figure 12. T-junction location update

2) Enter the surrounding road

In the location $\mathrm{B}$, that is, the vehicle in the cell door, determine whether you can turn right into the surrounding roads.

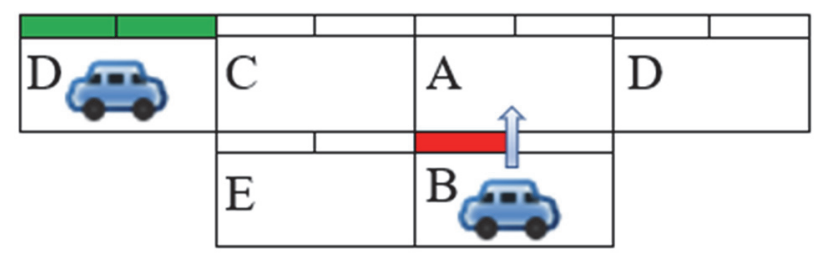

Figure 13. Ready to leave the district

a. If there is no car in position $\mathrm{A}$ and position $\mathrm{C}$ at this time, then the car at position $\mathrm{B}$ will send a right turn signal, and if the position $\mathrm{D}$ has a car at this point, it will decelerate to the $\mathrm{C}$ position and let the car of position B turn right. 


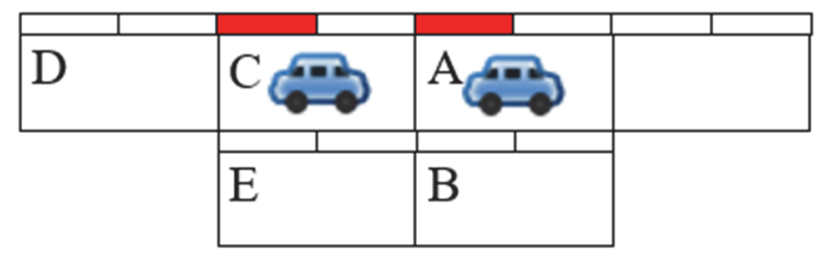

Figure 14. T-junction location update

b. If the position A does not have a car and the car at position $\mathrm{C}$ has issued a right turn signal, then the vehicle at position $\mathrm{B}$ sends a right turn signal and the vehicle at position $\mathrm{D}$ will decelerate.

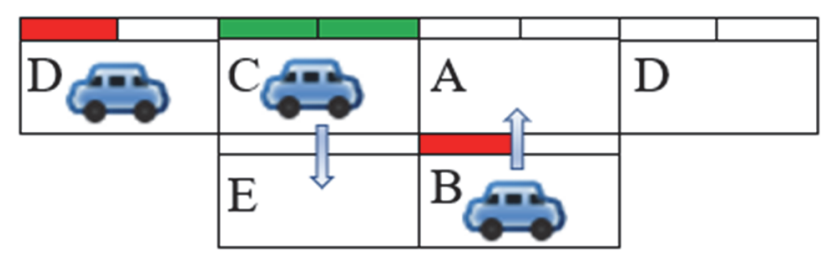

Figure 15. Ready to leave the district

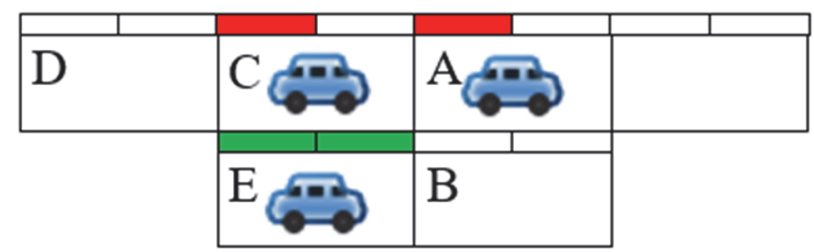

Figure 16. T-junction location update

Among them, the probability of right turn signal issue is based on Poisson distribution.

\subsubsection{The Sharing Rate of the Open District to the Surrounding Road Traffic Flow}

After the opening of the district, some of the vehicles on the surrounding roads will pass from within the district. The influence of the width of the road and the structure on the speed of the road cannot be neglected. However, this paper focuses on the change of the traffic capacity of the surrounding roads, thus, the concept of sharing rate, that is, the internal road of the district is abstracted, measure its share of traffic flow ratio by calculating its road area to the proportion of the total area of the surrounding road, which can make the model used more widely.

According to the "Urban Residential Area Planning and Design Code" published in 2002, the proportion of road in the district should be 9\% 17\% (Ministry of Construction of the People's Republic of China, 1994). However, actually, the land use of the residential area which includes some land for pedestrian or vehicle mix and the "broken head" road and so on, so convert the district's internal road into the surrounding road, the corresponding area:

$$
s_{x \rightarrow y}=s \times \gamma \times k
$$

Among them, $s_{x \rightarrow y}$ indicates the area within the district converted into the surrounding roads, $s$ indicates the actual area of the cell, $\gamma$ represents the proportion of the land area of the cell, and $k$ represents the proportion of the part that can be allowed to pass the external vehicle

Convert the district internal road into the surrounding roads using the above formula, use the proportional relationship to calculate the external traffic flow rate directly:

$$
\theta=\frac{s_{x \rightarrow y}}{s_{y}+s_{x \rightarrow y}}
$$

Here, $\theta$ represents the sharing rate of the traffic path, $s_{y}$ indicates the area of the surrounding road, and $s_{x \rightarrow y}$ expresses the area of the internal road which is converted into the area of the external main road. 


\section{Results and Discussion}

\subsection{The Effect of Different Types of the Opening Residential Area}

\subsubsection{Construct Different Types of the Residential Area}

In the study of the effect of the traffic capacity of the surrounding area after opening the closed residential area, the division of the type of the residential area is not the internal structure, but rather the residential area and the surrounding traffic as a whole, which shows a large difference in the city, suburbs and rural areas. Therefore, this paper draws on the urban, suburban and rural areas of Chengdu as a blueprint, and takes the average of the residential area of the same area as the residential area of the area to construct three types of residential area. In this paper, the residential areas are abstracted as rectangles, which the aspect ratio is golden ratio. The data of the constructed residential areas are shown in Table 2

Table 2. Construct Basic Information for Different Types of the Residential Areas

\begin{tabular}{cccc}
\hline Residential Area Type & Area & Length & Width \\
\hline Urban & 34602 & 237 & 146 \\
Rural & 54648 & 297 & 184 \\
Suburban & 109200 & 420 & 260 \\
\hline
\end{tabular}

According to the requirements for road area ratio within the residential area, this paper take its value of 0.13 . Based on design specifications of different levels of roads, we define the width of each lane is 3.5 meters, and set the surrounding road of the residential area to two-way lane. So we can get the area of the inner lane and the surrounding main lane on the residential area, and the traffic mode share of the residential area to the main road lane. The results are shown in Table 3.

Table 3. The Surrounding Area of the Residential Area and the Traffic Mode Sharing

\begin{tabular}{cccc}
\hline the Area of Residential Area & Urban & Rural & Suburban \\
\hline Area of Available Roads & 1349 & 2132 & 4258 \\
Area of surrounding roads & 2730 & 3416 & 4809 \\
Traffic Mode Sharing & 0.3307 & 0.3843 & 0.4696 \\
\hline
\end{tabular}

\subsubsection{Simulation Results of the Three Types of Residential Area}

This paper takes the early peak as the research perspective, and uses the improved NS model which is based on cellular automata to simulate three types of residential areas with MATLAB software. We get the value of the relevant index of the three residential areas before and after opening. The results are shown in Table 4.

Table 4. The Value of the Relevant Index of the Three Residential Areas

\begin{tabular}{|c|c|c|c|c|}
\hline Type & Index & Before Opening & After Opening & $\begin{array}{l}\text { Percent of } \\
\text { Improvement }\end{array}$ \\
\hline \multirow{4}{*}{ Urban } & $\begin{array}{l}\text { Average Passing } \\
\text { Speed }\end{array}$ & 33.75 & 35.25 & $4.30 \%$ \\
\hline & Saturation & 0.41 & 0.40 & $1.73 \%$ \\
\hline & Traffic Density & 0.16 & 0.17 & $-3.85 \%$ \\
\hline & $\begin{array}{l}\text { Accumulation of } \\
\text { Passing Time }\end{array}$ & 17353 & 14932 & $13.95 \%$ \\
\hline Suburban & $\begin{array}{l}\text { Average Passing } \\
\text { Speed }\end{array}$ & 34.78 & 36.13 & $3.88 \%$ \\
\hline
\end{tabular}




\begin{tabular}{ccccc}
\hline & Saturation & 0.39 & 0.38 & $2.56 \%$ \\
& Traffic Density & 0.15 & 0.16 & $-6.67 \%$ \\
& $\begin{array}{c}\text { Accumulation of } \\
\text { Passing Time }\end{array}$ & 20626 & 19257 & $6.64 \%$ \\
\hline \multirow{4}{*}{ Rural } & Average Passing & 34.83 & 36.17 & $4.09 \%$ \\
& $\quad$ Speed & 0.4 & 0.39 & $1.76 \%$ \\
& Saturation & 0.17 & 0.16 & $1.50 \%$ \\
& Traffic Density & 16794 & $6.53 \%$ \\
\hline & Accumulation of & 17967 & & \\
\hline
\end{tabular}

Based on Table 4, average passing speed, saturation and accumulation of passing time of the three different types of the residential area have been improved in different degrees after opening. Thanks to some vehicles can enter the residential area, the surrounding roads become smoother, thereby increasing the average speed of the vehicle; In addition, it has a diversion of traffic on the surrounding roads, which is reducing its actual traffic, thereby reducing its saturation and the passage of time on it. In addition to the residential areas of rural, the traffic density of surrounding roads are increased in urban and suburban after opening, it is due to there are more owners in the residential areas of urban and suburban, there will be more vehicles drive out of the residential area, leading to the vehicle is more intensive at the exits.

According to the fuzzy comprehensive evaluation, we get the value of Score of different types of residential areas after calculation. The results are shown in Table 5.

Table 5. The Value of Score of Different Types of Residential Areas

\begin{tabular}{cccc}
\hline Type & Urban & Suburban & Rural \\
\hline Score & $8.23 \%$ & $4.27 \%$ & $4.90 \%$ \\
\hline
\end{tabular}

Based on Table 5, we can see that the traffic capacity of surrounding roads of the residential areas are improved different after opening in different geographical. Among them, urban residential area has the best effect in it, which is up to $8.23 \%$. In the city, the traffic of surrounding roads of the residential area are surge in the rush hours, it can share the traffic on the surrounding roads, whereby easing the traffic pressure significantly after opening internal driveways of the residential areas. The value of Scores of the residential areas of suburban and rural are $4.27 \%$ and $4.90 \%$, which are lower than urban. It is because of the traffic less in suburban and rural, which are more open before opening.

\subsection{The Influence of Surrounding Road Structure on the Opening Effect of Community}

This paper takes the urban community as an example to study the change of the capacity of surrounding roads before and after the opening of the community under different lane numbers. Referring to China's "Design Criteria for Residential Areas", it can be seen that the road around the community exit may be 2 lanes to 8 lanes. According to the formula of sharing rate, the traffic sharing rate of surrounding roads after opening the urban community under different lane numbers can be calculated, as shown in following table 6 .

Table 6. Traffic Sharing Rate of Urban Community under Different Lanes Count

\begin{tabular}{ccccc}
\hline & Lanes Count & Urban & Suburban & Rural \\
\hline \multirow{3}{*}{ Traffic Mode } & 2 Lanes & 0.3307 & 0.4696 & 0.3843 \\
Share & 4 Lanes & 0.1953 & 0.3047 & 0.2353 \\
& 6 Lanes & 0.1372 & 0.2244 & 0.1682 \\
& 8 Lanes & 0.1049 & 0.1768 & 0.1301 \\
\hline
\end{tabular}

According to the improved NS model based on cellular automata, we use the MATLAB software to simulate different lanes. The results are shown in Table 7 and Table 8. 
Table 7. Evaluation indicators when the surrounding road structure changes

\begin{tabular}{ccccccccc}
\hline \multirow{2}{*}{ Lanes Count } & \multicolumn{3}{c}{ Before opening the community } & \multicolumn{4}{c}{ After opening the community } \\
\cline { 2 - 9 } & Lanes2 & Lanes4 & Lanes6 & Lanes8 & Lanes2 & Lanes4 & Lanes6 & Lanes8 \\
\hline Average speed & 33.75 & 34.56 & 34.56 & 34.83 & 35.37 & 35.1 & 34.83 & 35.37 \\
saturation & 0.4 & 0.42 & 0.43 & 0.42 & 0.4 & 0.42 & 0.42 & 0.42 \\
Traffic Density & 0.16 & 0.17 & 0.17 & 0.17 & 0.16 & 0.17 & 0.17 & 0.17 \\
Total transit & 17353 & 38490 & 61847 & 85431 & 14932 & 35776 & 57945 & 79353 \\
time & & & & & & & &
\end{tabular}

When the lanes count is lanes 2 , the effect of improving the surrounding road capacity after opening the community is more obvious, reaching $8.23 \%$. This is due to the fact that under this condition, the number of lanes is small, and the internal roads in the community can be diverted in time, thereby effectively alleviating the congestion of surrounding roads. However, the improving effects of lanes 4, lanes 6 and lanes 8 are not satisfactory. This is because when increasing lanes count continually, the traffic volume generated per hour remains constant, so that the proportion of traffic in each lane will decrease, that is, the traffic conditions of the roads around the community will be better. If the re-opening of the community is carried out at this time, there will be little effect.

Table 8. Percentage Improvement of Evaluation Indicators Before and After Opening Community

\begin{tabular}{rcccc}
\hline Lanes Count & Lanes 2 & Lanes 4 & Lanes 6 & Lanes 8 \\
\hline$\Delta$ Score & $8.23 \%$ & $4.06 \%$ & $3.75 \%$ & $4.02 \%$ \\
\hline
\end{tabular}

When the lanes count is lanes 2 , the effect of improving the surrounding road capacity after opening the community is more obvious, reaching $8.23 \%$. This is due to the fact that under this condition, the number of lanes is small, and the internal roads in the community can be diverted in time, thereby effectively alleviating the congestion of surrounding roads. However, the improving effects of lanes 4, lanes 6 and lanes 8 are not satisfactory. This is because when increasing lanes count continually, the traffic volume generated per hour remains constant, so that the proportion of traffic in each lane will decrease, that is, the traffic conditions of the roads around the community will be better. If the re-opening of the community is carried out at this time, there will be little effect.

\section{Conclusion}

To some historic reasons, the concept of gated communities has been widely accepted in the society, which makes the public ignore that the private road inside districts has become a stubborn obstacle in the development of urban road network. The concept of opening community will play a role as "blood capillary" in urban transportation network. From the macro perspective, it is undoubtedly an important measure to improve the urban living standards, which is an important measure to improve the quantity of city life. This paper studies the influence of the opening of the district on the capacity of the surrounding roads to evaluate the effect of the opening. First of all, we select a number of important traffic congestion indicators, and establish a comprehensive evaluation index system. Then, we improved the classic NS model, establish the changing-lane rules and the non-control T-insertion traffic rules, the and put forward the concept of sharing rate. Finally, we choose the city districts of different geographical types and different peripheral road structures in Shanghai as a case. Through analysis, simulation and comparison, we find that it is feasible to build up opening communities.

As the opening of districts will inevitably have some impact on residential owners, we will also evaluate the effect of residential opening with non-traffic indicators, such as mass satisfaction and economic benefits in the future research. In addition, this paper sets the probability of stochastic slowdown as constant, thus we ignore the fact that the moderating probability will increase with the increase of vehicle flow rate. Therefore, we can find the inherent relationship between the moderating probability and traffic flow through simulation and fitting, resulting that the model is more practical.

In this paper, we improve the classical Cellular Automaton NS model, consider the driving behavior of car overtaking, and study the traffic situation of T-junction in detail and finally get the model of the surrounding road traffic problems before and after the complete residential area is opened. Furthermore, we also put forward the concept of sharing rate, abstract the inner road of the district and creatively combine the district and the surrounding road into a whole. Therefore, the model built in this paper can be widely extended to all kinds of complex internal structure of the districts and undoubtedly, the effect of the opening of the community has practical reference 
significance.

\section{References}

Gao, X., \& Zhang, N. (2006). Advantages and disadvantages of closed communities and their countermeasures. Shanxi Architecture, 32(19), 34-35. https://doi.org/10.3969/j.issn.1009-6825.2006.19.022

Li, J., \& He, L. (2007). The Close and Openness of Residential Quarters: Interpretation of the "Central Garden" and "Fenghua Tiancheng" Residential Quarters. New Construction, (1), 93-96. https://doi.org/10.3969/j.issn.1000-3959.2007.01.021

Li, J., Qu, D. Y., Liu, C., Wang, J., \& Xu, X. (2016). Research on vehicle lane change behavior based on cellular automata. Highway Communications Science and Technology, 33(11), 140-145. https://doi.org/10.3969/j.issn.1002-0268.2016.11.021

Li, X. (2014). Urban Traffic Congestion Countermeasures - Closed Residential Area Traffic Opening Research. (Doctoral dissertation, Changsha University of Science and Technology). https://doi.org/10.7666/d.Y2756112

Li, X., Zhu, C. B., Li, D. M., \& Chen, K. (2017). Study on the Impact of Community Opening on the Capacity of Surrounding Roads. Journal of Hebei North University: Natural Science Edition, 33(9). https://doi.org/10.3969/j.issn.1673-1492.2017.09.008

Ministry of Construction of the People's Republic of China (1994). Planning and Design Code for Urban Residential Areas. China Building Industry Press.

Peng, L., Wu, D. Y., Liu, G. Q., \& Liu, M. R. (2005). Statistical decoupling of ns model of one-dimensional traffic flow cellular automata. Guangxi Science, 12(1), 35-38. https://doi.org/10.3969/j.issn.1005-9164.2005.01.010

Pucher, J., \& Dijkstra, L. (2003). Promoting safe walking and cycling to improve public health: lessons from the netherlands and germany. American Journal of Public Health, 93(9), 1509.

Qin, G. (2010). Study on the coordination between urban closed residential community planning model and urban transportation development. (Doctoral dissertation, Chang'an University). https://doi.org/10.7666/d.Y1729948

Wang, S. D., \& Liu, Y. (2015). Evaluation of land damage degree in mining areas based on improved fuzzy comprehensive evaluation model. Chinese Journal of Eco-Agriculture, 23(9), 1191-1198. https://doi.org/10.13930/j.cnki.cjea.150149

Yu, Y., \& Li, Z. T. (2006). "Open Block" Planning Concept and Its Implications for Urban Housing Construction in China. Planner, 22(2), 101-104. https://doi.org/10.3969/j.issn.1006-0022.2006.02.030

Zhai, P. (2004). Cancer in Urban Living: Problems and Countermeasures in Closed Residential Districts. Times Architecture, (5), 46-49. https://doi.org/10.3969/j.issn.1005-684X.2004.05.011

Zou, Y., \& Yan, H. N. (2000). Reflections on the mode of urban residential communities in China. World Architecture, (5). https://doi.org/10.16414/j.wa.2000.05.002

\section{Copyrights}

Copyright for this article is retained by the author(s), with first publication rights granted to the journal.

This is an open-access article distributed under the terms and conditions of the Creative Commons Attribution license (http://creativecommons.org/licenses/by/4.0/). 